\title{
Premalignant and neoplastic skin lesions associated with occupational exposure to "tarry" byproducts during manufacture of 4,4'-bipyridyl
}

\author{
G T BOWRA, ${ }^{1}$ D P DUFFIELD, ${ }^{1}$ A J OSBORN, ${ }^{1}$ AND I F H PURCHASE ${ }^{2}$ \\ From Imperial Chemical Industries Ltd, Mond Division, ${ }^{1}$ Runcorn, and Central Toxicology Laboratory, ${ }^{2}$ \\ Alderley Park, Macclesfield, Cheshire, UK
}

ABSTRACT Skin lesions have been identified in 20 workers manufacturing 4,4'-bipyridyl. The lesions were on the face, neck, and backs of the hands and arms. Larger localised lesions were removed surgically and examined histopathologically, but other lesions have been treated topically with fluorouracil cream. Histological diagnosis showed a progression from keratosis to Bowen's disease and, in six cases, to squamous cell carcinoma. Over 550 individuals associated with 4,4'-bipyridyl processes between 1961 and 1980 have been examined or questioned. All 20 patients identified worked in now obsolete production plants; 15 of whom were found among the 147 shift process operators. The time taken for the lesions to appear from first working on a 4,4'-bipyridyl plant was 6-19 years (average 12 years). Starting in the early 1960s three processes have been successively used to convert pyridine into 4,4'-bipyridyl. These differ in the reaction conditions used and in the efficiency of the conversion. In the two earlier plants a substantial quantity of pyridine was converted into polypyridyls and "tarry" byproducts, which caused operating difficulties and increased the risk of worker exposure. The exposure and occupational histories of the cases, including the fact that two had worked only on the obsolete production plants, indicates that the causative agent was present in these earlier processes. In-vitro tests for potential carcinogenicity on materials from the present plant and from an earlier process gave positive tests on the tarry byproducts from the earlier process, suggesting that they were the cause of the lesions. Tar impurity production on the present plant is minimal, and worker exposure to chemicals is low. No similar skin lesions have been seen in those who have worked only on the present plant. This observation supports the view that the causative agent was present only on the earlier plants but is not conclusive because of the shorter time that has elapsed since first exposure of personnel who have worked only on the present plant. Medical surveillance of past and present 4,4'-bipyridyl workers is continuing.

During 1976 several shift process operators in the 4,4'-bipyridyl plant at Widnes were found to be suffering from various skin lesions on the hands, forearms, and sometimes the scalp. We describe the comprehensive study to identify the extent of this problem and the probable cause and the continuing health surveillance programme.

\section{Methods}

IDENTIFICATION OF POPULATION AT RISK In 1976 all 158 personnel working in the plant (which manufactures 4,4'-bipyridyl for use in paraquat Received 8 June 1981 Accepted 5 August 1981 production) were examined clinically by the work's medical officer. Twelve shift process operators were referred to a consultant dermatologist, who confirmed that 11 had skin lesions described as keratoses, including one with an epithelioma. As all patients had also worked on the previous 4,4'-bipyridyl plants, it was decided to follow up all personnel who had worked on the plants since manufacture started in 1961.

The names of all workers associated with manfacture of 4,4'-bipyridyl or its subsequent conversion to paraquat and the packing of paraquat solutions were obtained from employee records and personal recall by those working in various jobs on different units. Individuals were traced from personnel files, 
pension records, voters' lists, and personal contact. Two discontinued processes (high temperature sodium (HTS) and magnesium (MAG)) and one existing process (low temperature sodium (LTS)) have been used to manufacture $4,4^{\prime}$-bipyridyl at Widnes. Little information on quantitative occupational hygiene is available from the early plants, but descriptions of the processes and conditions of work were obtained from plant personnel who knew of conditions on all three plants.

\section{CLINICAL AND MORTALITY STUDIES}

All those traced were offered a medical examination, but a few said that they had no skin problems and declined it. They were classified as "informed with no skin lesions" (table 1). Anyone with lesions on the skin was referred to a dermatologist. Treatment included surgical removal of larger isolated lesions and the application of fluorouracil cream to other lesions.

The company operates a computerised system for recording and analysing absences from work of 28 days or longer, which has been operating since 1975. The absences of employees on the present plant have been examined.

All workers who had died have been traced from personnel and pension records and death certificates. The causes of death were established from the death certificates or medical records and these were compared with the expected number calculated from mortality statistics of England and Wales.

\section{OCCUPATIONAL RECORDS}

Each worker was listed under one job category only, this being the category in which the person had either the longest service or the greatest exposure to chemicals on the plants. They were then grouped according to the plant or combination of plants on which they had worked. The number of workers classified as traced, not traced, and dead was obtained from the list of workers and medical records.

A more detailed survey of shift process operators was undertaken. Data on the plants on which they worked, the period they had worked on the plants ("service times"), the period since first starting work on a 4,4'-bipyridyl plant ("elapsed time") (table 2) and the time between starting working on the plant and the appearance of lesions ("years to onset from first exposure") (table 3) were obtained from the medical and occupational records.

In an attempt to look for a link between skin disorders and a particular plant area or job the work histories of 24 plant shift operators who worked on both the MAG and the LTS processes were analysed in detail to obtain the period worked in the various areas of the plant.

INVESTIGATING SUBSTANCES ON THE PLANTS FOR EVIDENCE OF CARCINOGENICITY AND MUTAGENICITY

A list of 99 identifiable chemicals used in the three processes was prepared, and publications and company records were searched to identify any substances with known carcinogenic properties.

Representative process chemicals were tested for potential carcinogenicity in the Salmonella reverse mutation assay ${ }^{1}$ and cell transformation assay. ${ }^{23} \mathrm{~A}$ laboratory rig was set up to produce samples by the discontinued MAG process and samples were selected from the current LTS process.

HEALTH MONITORING

A continuing health monitoring programme was

Table 1 Summary of survey for skin lesions in workers grouped by job categories as at 31 December 1980

\begin{tabular}{|c|c|c|c|c|c|c|}
\hline & No in group & No examined & $\begin{array}{l}\text { No informed } \\
\text { with no skin } \\
\text { lesions }\end{array}$ & Not traced & Dead & $\begin{array}{l}\text { No of cases in } \\
\text { group }\end{array}$ \\
\hline Shift process operators & 147 & $118(8$ dead $)$ & $9(1$ dead $)$ & 9 & 20 & 15 \\
\hline Day process operators & 30 & 26 & 3 & - & 1 & - \\
\hline Women product packers & 23 & 22 (1 dead) & 1 & - & 1 & - \\
\hline Plant supervisors & 15 & 13 & - & - & 2 & - \\
\hline Plant laboratory staff & 50 & 35 (1 dead) & 6 & 8 & 2 & 1 \\
\hline Technical and administrative staff & 74 & 71 ( 2 dead $)$ & 3 & - & 2 & 1 \\
\hline \multicolumn{7}{|l|}{ Engineering maintenance } \\
\hline (fitters, pipefitters) & 89 & 82 ( 3 dead $)$ & 2 & 1 & 7 & 2 \\
\hline \multicolumn{7}{|l|}{ Miscellaneous trades maintenance } \\
\hline (boilermakers, plumbers, laggers) & 56 & 49 & 1 & 6 & - & 1 \\
\hline Instrument maintenance & 80 & 56 & 12 (1 dead) & 12 & 1 & - \\
\hline Electrical maintenance & 24 & 22 & 1 & 1 & - & - \\
\hline \multicolumn{7}{|l|}{ Research and development staff } \\
\hline (laboratory and semitechnical plants) & 41 & 23 & 6 & 11 & 1 & - \\
\hline Total & 629 & 517 ( 15 dead $)$ & 44 ( 2 dead $)$ & 48 & 37 & 20 \\
\hline
\end{tabular}

"No informed with no skin lesions" is the number of people who have been informed about the skin disorder and have no skin lesions; however, they have not attended a company medical examination. 
instituted in mid-1977 for all past and present 4,4'bipyridyl workers. All workers traced, including contacted at 4-12 month intervals, depending on their those who have retired or left the company, are plant/job category, and offered clinical appointments. Shift process operators and other highly exposed

Table 2 Survey of shift process operators showing elapsed times and duration of service on various plants as at 31 December 1980

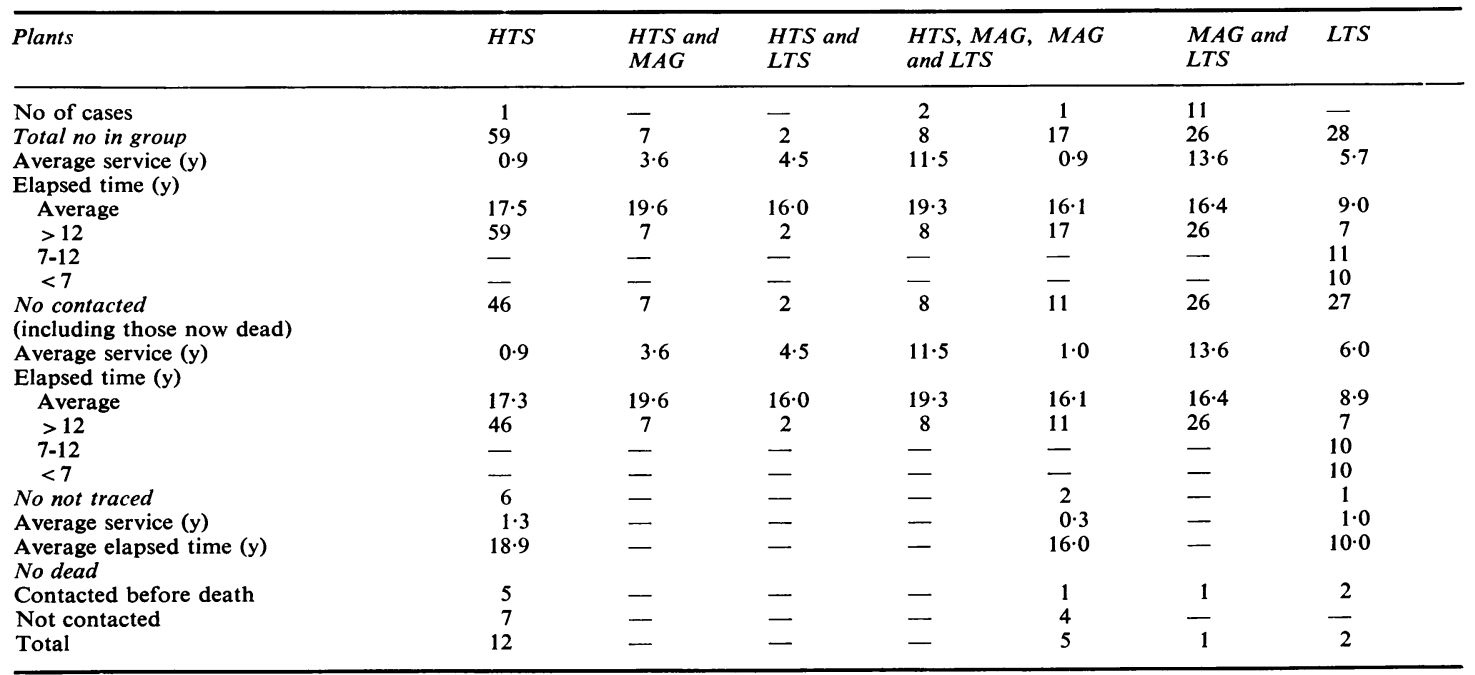

No contacted $=$ Number "examined" plus the number "informed with no skin lesions."

HTS = High temperature sodium

MAG = Magnesium.

LTS $=$ Low temperature sodium.

Table 3 Clinical information on 20 4,4'-bipyridyl workers with occupationally induced skin lesions as at 31 December 1980

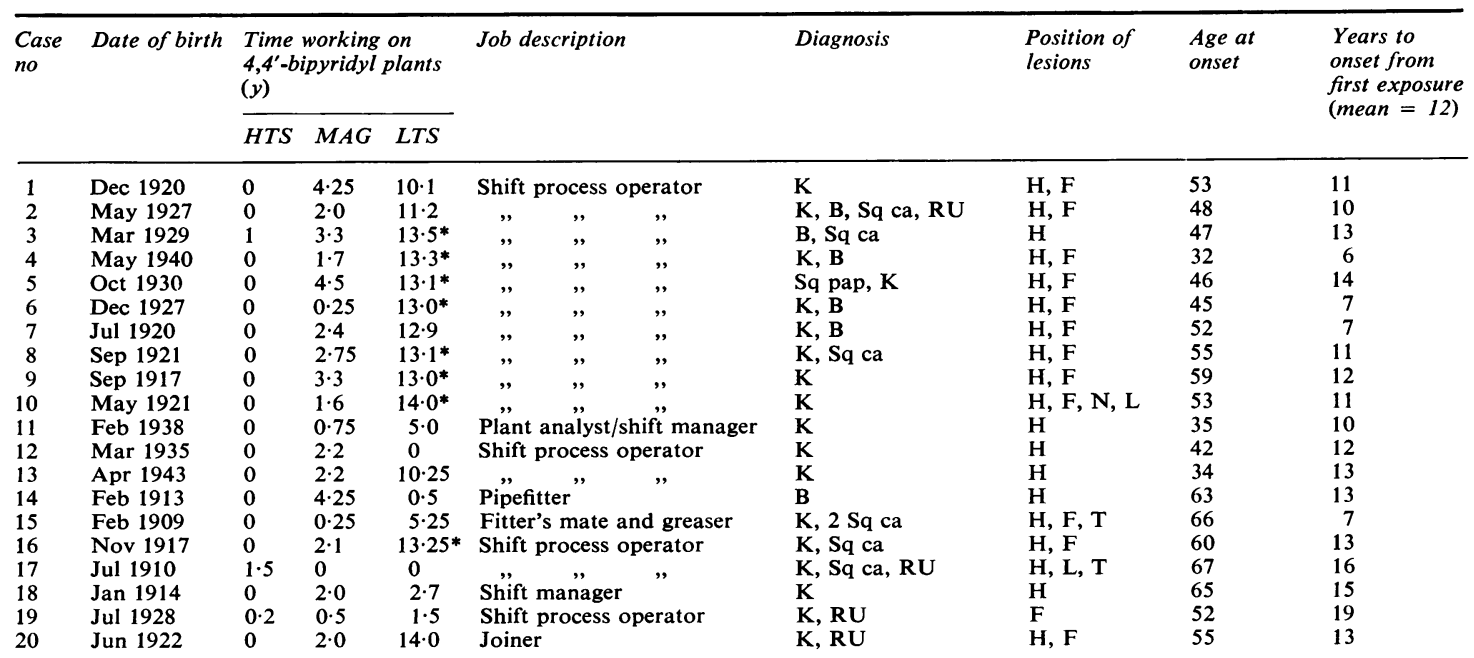

All diagnoses, except for case 1, have been confirmed histologically. Patient 2 has died of an unrelated illness. *Continuing to work on LTS plant.

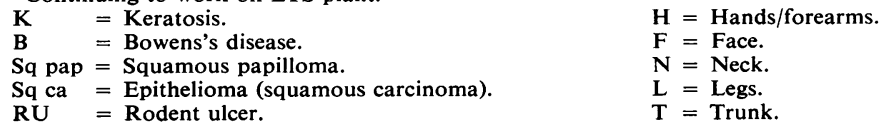


groups from the MAG plant are called in every four months, less exposed groups from the MAG plant every eight months, and all others, including HTS and LTS plant workers, every 12 months.

\section{Results}

POPULATION AT RISK

At the end of 1980 , the position with respect to the 629 people listed as having worked on the development, commissioning, and operation of $4,4^{\prime}$ bipyridyl plants was as follows: 581 had been traced, of whom 37 were dead and 20 had skin lesions. Most of the 48 workers not traced were in the instrument maintenance and research and development staff categories, where turnover of personnel was above average. Exposure to chemicals by workers in these categories was relatively low.

\section{MANUFACTURING PROCESS AND}

OCCUPATIONAL EXPOSURE

Three processes have been used to produce $4,4^{\prime}$ bipyridyl at Widnes. The chemistry used in the three processes is essentially the same in that pyridine is dimerised to a reduced species that is then oxidised to 4,4'-bipyridyl.

The reaction conditions used and efficiency of conversion differ, however, and hence the nature and quantities of the resultant byproducts differ. After isolation the 4,4'-bipyridyl is quaternised with methyl chloride (also dimethyl sulphate in 1961-9) to give a 1,1'-dimethyl-4,4'-bipyridylium (paraquat) salt (fig).
The magnesium process plant (1962-7) also consisted of several batch operations; there were more stages and more recycling of materials. The conversion efficiency of pyridine to 4,4'-bipyridyl was somewhat higher than in the HTS plant, but a considerable amount and variety of byproducts such as bipyridyl isomers, terpyridyls, polypyridyls, and large amounts of similar insoluble tars were formed.

Exposure for both process operators and maintenance personnel was much higher than on the HTS plant, mainly due to process difficulties. There was additional handling of intermediate chemicals. Tarry deposits formed blockages in pipework and equipment, which often had to be dismantled to remove the tars by using steam lances and rodding equipment. These activities and the batch nature of the process led to relatively poor conditions of hygiene. Skin contact with the non-corrosive process materials was a problem, and there was exposure to vapours.

The low temperature sodium plant, which has operated since 1966, converts pyridine to $4,4^{\prime}$ bipyridyl with almost $100 \%$ efficiency. The process materials are well contained in a continuous, automated plant, mainly operated remotely from a control room. Exposure of process operators and maintenance personnel is low. Skin contact is minimal because the chemicals are not handled in the process, and protective equipment is worn during sampling and maintenance. In addition measured concentrations of process materials such as 4,4'bipyridyl and pyridine in the plant atmosphere are very low.

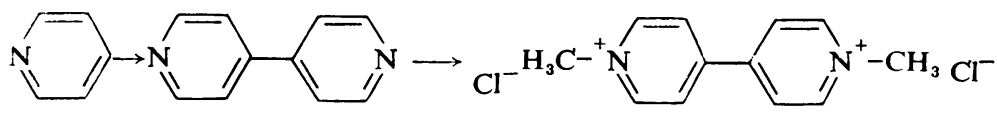

pyridine $\quad 4,4^{\prime}$-bipyridyl

The high temperature sodium plant (1961-3) was relatively small and consisted of several batch operations. In this process only $35 \%$ of the pyridine consumed was converted to 4,4'-bipyridyl, and substantial quantities of bipyridyl isomers, polypyridyls, and "tars"* were produced. Materials were contained in the plant apart from 4,4'-bipyridyl and "tarry" still residues, which were run molten from the stills into open drums. When solidified the 4,4'bipyridyl was broken up with sledge hammers. Process operators were exposed to fumes and to bipyridyl dust, but exposure of other groups of workers was low.

\footnotetext{
* The tars formed on the HTS and MAG plants were similar in appearance to coal or petroleum tars, but the composition was chemically different.
}

CLINICAL AND MORTALITY STUDIES

Table 3 provides a summary of the 20 cases identified. In 19 of these the larger isolated lesions were removed surgically and examined histopathologically.

Individual lesions have been diagnosed as keratoses, Bowenoid changes, Bowen's disease, rodent ulcer, squamous papilloma, and squamous carcinoma.

The severity has varied from isolated keratoses of $1 \mathrm{~mm}$ diameter to extensive skin involvement on the back of hands, forearms, face, and hairless areas of the scalp. In one case two isolated lesions occurred on parts of the body normally covered by clothing. There were no lesions observed on the palmar surface of the hands and no lesions requiring treatment on the flexor surface of the arms. 
Patients have been treated by surgical removal of larger isolated lesions. Other lesions have been treated with topical fluorouracil cream. Some of the patients have experienced a recurrence of lesions, or development of lesions in different areas, and further treatment has been necessary.

Analysis of the computerised system for the recording of absences from work of 28 days or longer failed to show any significant clusters of disease. It should be recognised that the system has been in operation for only six years, so that any excess would need to be quite striking in order to be detected.

The population contained three workers with a history of rodent ulcers and one with a malignant melanoma; none of these have keratoses, and they are not included in the list of cases. A statistical analysis of the 37 causes of death among the population surveyed showed no abnormal trends. The mortality from cancer (nine cases) showed no excess against expected numbers, and there was no unusual pattern. No diagnosis of skin cancer occurred on any of the death certificates.

OCCUPATIONAL RECORDS

A summary of the survey with workers grouped in job categories is given in table 1. Each person is listed under one category only, this being the category in which the person had either the longest service or the greatest exposure to chemicals on the plants. Fifteen of the 20 cases were shift process operators, with three maintenance workers, one technical and one laboratory worker.

Table 3 shows a variation in the estimated period to onset of the skin disorder from first exposure associated with 4,4'-bipyridyl plant of from six to 19 years, with an average of 12 years. In many of the cases time of onset is only judged by individual worker recollection and therefore can only be approximate: keratosis may well have been present for some time before being noticeable. The effect of age on latent period was considered, but no significant correlation was found between "years to onset of disorder" and "age when first exposed."

A more detailed survey of the shift process operators is given in table 2. They are grouped according to the plant, or combination of plants, on which they worked. Twenty-six long-serving shift process operators worked on both the MAG and LTS plants, transferring to the LTS plant when the MAG plant was shut down. Most cases have occurred in this group. Of the remaining patients, one worked on the HTS plant only, one on the MAG plant only, and the other two worked on all three plants. The HTS plant was located at a separate works, and fewer men transferred from there to the MAG or LTS plants.
Average service times and elapsed times since first exposure on a 4,4'-bipyridyl plant are given for comparison with the information on cases given in table 3.

In an attempt to look for a link between the skin disorders and a particular plant area or job the work histories of 24 MAG and LTS plant shift operators were analysed in detail to obtain the period worked in various areas of the MAG and LTS plants. Most operators had worked in various areas, and analysis showed no significant correlations.

\section{INVESTIGATION OF SUBSTANCES PRESENT IN} THE PLANTS

Six carcinogens or potential carcinogens were identified from the survey of publications, and five of these (benzene, hexavalent chromium, methyl chloride, methylene chloride, and dimethyl sulphate) were not known skin carcinogens, nor was there a history of skin contact. The sixth, mineral oil, has been associated with the induction of skin cancer in $\operatorname{man}^{4}$ and was used on all three plants for engineering purposes. Modern oils are solvent refined, reducing their contamination with carcinogens, and again there was no history of excessive skin contamination of the affected process operators. There were no other chemicals with potential carcinogenic properties, and for many (including paraquat) there was good evidence of the absence of carcinogenicity in suitable laboratory experiments.

Forty-four samples were tested in the Salmonella assay and 12 of them in the cell transformation assay. Unequivocally positive results were obtained with three samples to which the affected workers were exposed:

(a) A solvent extract containing tars and polypyridyl byproducts derived from the MAG process yielded five fractions that were positive in the Salmonella test.

(b) The unfractionated tars were toxic to Salmonella, giving negative results, but were positive in the cell transformation assay. The shift process operators on the MAG plant had been extensively exposed to these solvent and tar mixtures.

(c) 4,4'-bipyridyl was positive in the cell transformation, but not the Salmonella assay.

It was not likely to be the cause of the lesions since only one case occurred among HTS plant process operators, whose exposure to $4,4^{\prime}$-bipyridyl was considered to be highest of the workers on the three processes. Nevertheless, a life-time mouse skin painting programme is being carried out to obtain further information on the toxicity of 4,4'-bipyridyl.

Negative results were obtained in both assays for other chemicals to which the workers were exposed. In particular, paraquat is negative in both in-vitro 
assays and in long-term carcinogenicity assays. The workers who pack paraquat and spray operators have been examined, and no evidence of similar skin lesions has been found. ${ }^{5}$

\section{Discussion}

The keratoses and other lesions seen in the 20 workers are clinically and pathologically indistinguishable from those produced in fair-skinned people by prolonged exposure to sunlight (solar keratosis), or occurring in the elderly (senile keratosis). The pattern of incidence and the clinical picture have led us to assume that the lesions are all part of a single clinical entity. The incidence (20 cases out of 561 4,4'-bipyridyl workers contacted; 15 cases out of 127 shift process operators contacted) is excessive for a group of workers in the north-west of England. This is confirmed by the absence of cases among other groups of workers in the survey (table 1). It is therefore concluded that the lesions are occupationally induced.

There was no evidence from the cause of death of the 37 dead workers to suggest that any fatal occupationally induced disease was present in this group of workers.

Although 48 workers were not traced, they were predominantly from the groups with low and short exposure and thus were less likely to be suffering from occupationally induced disease.

All but two of the cases had worked on two or more of the 4,4'-bipyridyl processes. One had worked only on the HTS plant, the other only on the MAG plant, and the remaining 18 on a combination of plants including either the MAG or HTS plants. This indicates that the causative agent was present in these earlier and now obsolete plants. No cases have occurred among over 300 personnel who have worked only on the LTS plant. This observation supports the view that the causative agent was present only on the earlier plants but is not conclusive because of the shorter elapsed times of personnel who have worked only on the LTS plant.

The descriptions of the working conditions on the three plants provide evidence that exposure to chemicals was much greater on the HTS and MAG plants, with shift process operators on the MAG plant being particularly heavily exposed during clearing of blockages. Exposure on the LTS plant is much lower. These patterns of exposure are compatible with the conclusions from the occupational histories that the causative agent(s) was in the HTS and MAG plants.

The lesions have occurred predominantly among shift process operators with $75 \%$ of cases in this group. From table 2 it may be seen that there are 11 cases in 26 shift process operators who worked on both the MAG and LTS plants, but only one case in 18 who worked on the MAG but not the LTS plants.

The most likely explanations for the lower incidence in the group of 18 is that they had low service times on the MAG plant, part of which was training, and almost all then left the company. By contrast, the group of 26 who transferred to the LTS plant had been the most exposed group on the MAG plant.

The survey of publications on the potential carcinogenicity of the chemicals used on the plants failed to identify any chemicals that could have caused the lesions. In-vitro assays carried out on various samples from the MAG and LTS processes have identified a solvent extract, used in the MAG process, that contained polypyridyls and tars, with activity indicative of potential carcinogenicity.

The information from occupational histories, occupational exposure descriptions, and the in-vitro test led to the conclusion that the substance most probably responsible for the skin lesions was the tarry byproduct present in the solvent extract from the MAG process. Similar tarry deposits were present in the HTS, but not the LTS, process, and exposure to these deposits is presumed to be the cause of the skin lesions in the HTS process operator. None of the other chemicals, including paraquat, is a likely cause of the skin lesions. Since the MAG and HTS processes have been discontinued, it seems unlikely that the substance that caused the lesions will ever be conclusively established. Nevertheless, it is clearly prudent to take steps to minimise exposure to chemicals in all other processes for making 4,4'bipyridyl or that are likely to produce polypyridyls, and to continue to monitor the health of the workers.

We thank S O'Brien, P B Dransfield, E Longstaff, J Ashby, J A Styles, and G M Paddle for their help in this project.

\section{References}

1 Ames BN, McCann J, Yamasaki E. Methods for detecting carcinogens and mutagens with the Salmonella/mammalian microsome mutagenicity test. Mutat Res 1975; $31: 347-63$.

2 Styles JA. A method for detecting carcinogenic organic chemicals using mammalian cells in culture. $\mathrm{Br} J$ Cancer 1977;36:558-63.

${ }^{3}$ Purchase IFH, Longstaff E, Ashby J, et al. An evaluation of six short term tests for detecting organic chemical carcinogens. Nature 1976;264:624-7.

${ }^{4}$ Kipling MD. Oil and cancer. Ann $R$ Coll Surg Engl 1974; 55:71-9.

5 Howard JK. A clinical survey of paraquat formulation workers. Br J Ind Med 1979;36:220-3. 